\begin{tabular}{|l|l|l|}
\hline \multicolumn{2}{|c|}{ PublisherInfo } \\
\hline \hline PublisherName & $:$ & BioMed Central \\
\hline \hline PublisherLocation & $:$ & London \\
\hline \hline PublisherImprintName & $:$ & BioMed Central \\
\hline \hline
\end{tabular}

\title{
Active compression-decompression improves outcome after cardiac arrest
}

\begin{tabular}{|l|l|l||}
\hline \multicolumn{2}{|c||}{ ArticleInfo } \\
\hline \hline ArticleID & $:$ & 4099 \\
\hline \hline ArticleDOI & $:$ & $10.1186 /$ ccf-1999-1881 \\
\hline \hline ArticleCitationID & $:$ & 1881 \\
\hline \hline ArticleSequenceNumber & $:$ & 36 \\
\hline \hline ArticleCategory & $:$ & Paper Report \\
\hline ArticleFirstPage & $:$ & 1 \\
\hline \hline ArticleLastPage & $:$ & 4 \\
\hline \hline & & RegistrationDate : 1999-9-27 \\
\hline ArticleHistory & $:$ & OnlineDate \\
\hline \hline ArticleCopyright & $:$ & Current Science Ltd1999-9-27 \\
\hline \hline ArticleGrants & $:$ & \\
\hline \hline ArticleContext & $:$ & 130541111 \\
\hline \hline
\end{tabular}




\section{Keywords}

Advanced life support, cardiac arrest, cardiopulmonary resuscitation

\section{Comments}

Resuscitation after cardiac arrest is a difficult field in which to perform controlled trials and this study is a highly impressive collaborative effort.

The beneficial effect of ACD may have been diluted by the fact that patients were actively assigned to ACD or standard CPR for only advanced life support. Only 99 patients received standard CPR for both basic and advanced life support and only 120 received ACD for both parts of resuscitation.

Nevertheless, the beneficial effect of ACD on one year survival only just reaches statistical significance (lower $95 \%$ CI for odds ratio $=1.03$ ). It is a pity that patients were not randomised as the criteria for exclusion leave some room for judgement. Bias would only have to account for a small number of the excess deaths in the standard CPR group to result in a type I error. More than 20 patients in each group received the wrong method of advanced life support and it would be interesting to know whether on an actual treatment (rather than the intention to treat analysis presented), there was still a significant difference in one year survival.

More detailed information on neurological status at 1 year compared with say $72 \mathrm{~h}$ would be interesting for intensivists, who often face difficult treatment decisions in immediate survivors of cardiac arrest. The 5\% long-term survival in the ACD group is impressive, but caution should be exercised in extrapolating these results to countries with immediate care arrangements that do not involve physicians. Intensivists will note that there were no one year survivors for nonwitnessed arrests but that the neurological status of survivors of witnessed arrest was good.

In view of the previous trials which have found no benefit of ACD, it might be wise to obtain further data before other countries invest in the high level of training and expertise that would be required to introduce ACD into protocols for out-of-hospital arrest.

\section{Introduction}

Active compression-decompression (ACD) using a chest wall suction device increases venous return during the decompression phase compared with standard cardiopulmonary resuscitation (CPR). 
Although a number of trials haveshown no benefit of ACD over standard CPR, two studies have shown an improvement in short term survival with ACD.

\section{Aims}

To determine whether one-year survival and neurologic outcome following out-of-hospital cardiac arrest are better when ACD is used during advanced life support.

\section{Methods}

Patients suffering out-of-hospital arrest in the study area were assigned to standard CPR (377 patients) or ACD (373 patients) during advanced life support. Patients with presumed irreversible cardiac arrest, known terminal illness, a do-not-resuscitate order, who waited more than 30 minutes between arrest and basic life support or, who developed palpable pulses with basic life support were excluded.

\section{Results}

Survival at one year was $2 \%$ with standard CPR and 5\% with $\mathrm{ACD}$, odds ratio $2.5(95 \% \mathrm{CI}=1.03$ 6.2). Hospital discharge without neurologic impairment was $2 \%$ with standard CPR and $6 \%$ with ACD, odds ratio $3.2(95 \% \mathrm{CI}=1.3-7.5)$. There were also statistically significant improvements in survival at 7 days, $24 \mathrm{~h}$ and $1 \mathrm{~h}$ for ACD. Neurologic outcomes measured with the Glasgow-Pittsburgh cerebral performance categories were similar in the two groups, 1.5 versus 1.3 ( standard CPR versus ACD). The difference in number of one-year survivors in whom neurologic status returned to baseline was not statistically significant (12 of 17 for ACD compared with 3 of 7 for standard CPR).

Patient groups were well matched for baseline characteristics. All one-year survivors in both groups had a witnessed cardiac arrest.

\section{Discussion}

Survival at one year in the ACD group was more than twice that in the standard CPR group, and was higher than the survival rate following hospital discharge after standard CPR in other studies in metropolitan areas. More than half the ACD survivors had an initial rhythm of asystole. 
Subgroup analysis suggests that ACD may be particularly effective in patients presenting with asystole, in whom it might more effectively restore metabolic support for cardiac contractility.

The percentage of patients with asystole was high compared with that in other studies and the outcome of these patients was better than in other series.

The failure of other studies to show a benefit of ACD may be explained by differences in the quality of training, fatigue in resuscitation personnel, failure to monitor compression-decompression with the pressure gauge, and use of the ACD device in a moving ambulance. The protocol in this study paid very close attention to all these details.

France is different from the US, Canada and Britain in providing physician support at the scene of cardiac arrest. This study also differs from others in providing advanced life support for at least 30 minutes regardless of the presenting rhythm.

ACD CPR increases the patient's chance of short- and long-term survival and its use should be promoted after proper training of rescue personnel.

\section{References}

1. Plaisance P, Lurie KG, Vicaut E, Adnet F, Petit JL, Epain D, Ecollan P, Gruat R, Cavagna P, Biens J, Payen D: A comparison of standard cardiopulmonary resuscitation and active compressiondecompression resuscitation for out-of-hospital cardiac arrest. N Engl J Med. 1999, 341: 569-575. 